\title{
CONTRIBUCION A LA ECOLOGIA DE TIVELA MACTROIDES, BORN, 1778: ASPECTOS REPRODUCTIVOS
}

ANTULIO S. PRIETO A.

Departamento de Biologia, Escuela de Ciencias

Universidad de Oriente, Cumaná, Venezuela

\section{SYNOPSIS}

Sexual maturity and other reproductive features of Tivela mactroides collected from Playa Guiria, Carupano, Sucre State, Venezuela, were studied on the basis of smears and histological sections of ronads. There was continuous sexual cycle, but the develobment of the visceral mass, in females was less from January to March, 1975. A five steps maturity sexual scale was used to examine the histological sections, indicating that the dominant phase consist of a partial spawning. In total sexes and in intervals $(10.1-300 \mathrm{~mm})$ the ratio does not varv from the proportion 1:1, although the males outnumber the females; the number of females is significantly higher in the larger ones (30,1.45.00 mm).

\section{Introduccion}

Los estudios sobre aspectos ecológicos de bivalvos han revibido mucha atención.

La reproducción de los lamelibranquios y las características de la gametogénesis han sido objeto de intensivos estudios y se ha comprobado que la sexualidad puede variar con la edad, Yoloye (1970), Vélez (1975) y Caddy (1967).

En Venezuela. el estudio sobre la ecología de bivalvos, se ha orientado principalmente hacia especies comerciales entre las que podemos señalar el mejillón Perna perna (Vélez \& Martinéz, 1967 y Carvajal. 1969).

Tivela mactroides Born, 1778 es un pelecipodo perteneciente a la familia Veneridae que se distribuye desde las Indias Occidentales hasta Brasil; incluyendosele en los catálogos malacológicos de Sur América como una espécie de amplio rango de distribución en el trópico (Warmke \& Abbott, 1961). Narchi (1972) estudió las características anatómicas y funcionales del aparato alimentario de este bivalvo en playas de Brasil.

Debido al desconocimiento que se tiene de otros aspectos biológicos, hemos realizado este trabajo en el cual se estudian las características de las gónadas, madurez sexual y proporción de sexo.

\section{Materiales y Metodos}

Los ejemplares de Tivela mactroides fueron colectados mensualmente en Playa Guiria, localidad situada al oeste de la ciudad de Carúpano, Venezuela, desde Julio de 1974 hasta Junio de 1975. La gametogenesis, proporción de sexos y otros aspectos reproductivos se estudiaron por dos métodos diferentes:

1. Por examen macroscópico y microscopico de la masa visceral y las gónadas a una alicuota de la colecta.

Para ello se observaron bajo lupa y una porción de las mismas fue separada de la parte interna y examinadas al microscopio. El sexo fue determinado por la presencia de células germinales.

En las hembras se anotó la condición de maduración de acuerdo a lo sefralado en la Tabla I y en los machos se tomó en cuenta la abundancia de espermatozoides. Los resultados de madurez sexual y proporción de sexos se tabularon relacionandolas en intervalos de sexo y en clase de $5 \mathrm{~mm}$.

2. Por observación microscópica de cortes histológicos provenientes de gonadas fijadas : con Bouin en ejemplares colectados mensualmente con tallas que oscilaban entre $7 \mathrm{~mm}$ y $40 \mathrm{~mm}$ de longitud total distribuídos en rangos equitativos, y coloreados con hematoxilina ferrica de Mayer y eosina. Los cortes se realizaron a nivel medio de la masa visceral con un espesor entre 7 y 12 micras en un micrótomo. En las láminas se determinó el sexo y escala de madurez sexual siguiendo las recomendaciones de la literatura (Loosanoff, 1953, Boyden, 1971 y Caddy, 1967 ). En cada múestreo se determinó la salinidad y la temperatura del agua superficial utilizando un termómetro con precisión de $0,1^{\circ} \mathrm{C}$.

TABLA I. Grados arbitrarios de maduración gonadal asignado por - observación macroscópica y microscópica de frotis gonadal en hembras de Tivela mactroides colectadas en Playa Guiria.

GRADO 1 - Gónoda escasa y, poco desarrollada, flacida o aparentemente hueca. Espesor de la masa visceral mínimo, pueden existir algunos ovocitos. $\mathrm{Si}$ el ovario se encuentra en crecimiento hay ovocitos de diferentes tamaños peró la proporción de ovocitos jóvenes pendunculados y los pequeños es mayor. Probablemente representa estados muy avanzados de desove 0 de maduración incipiente.

GRADO II - Gónoda amplia, masa visceral y región del pie distendida. Lateraimente se observan las masas foliculares por debajo de la membrana que recubre la parte media del cuerpo. Los frotis presentan gran cantidad de ovocitos con talla de 95 micras, de forma piriforme o esférica. Estos presentan núcleos vesiculosos y al avanzar la maduración la membrana nuclear tiende a desaparecer. Pueden observarse ovocitos pequeños pero en menor proporción que los grandes. En este estado, la gónada esta desovando parcialmente o próxima a hacerlo.

\section{Resultados}

Los organos sexuales en Tivela mactroides se encuentran incluídos en la porción superior del cuerpo visceral, rodeando virtualmente al intestino y al estómago y limitados dorsalmente por los rifones y el hepatopancreas.

No se encontro dimorfismo sexual entre hembras y machos. En las hembras el producto gonadal es más granuloso y denso que en los machos.

En la Figura 1, se indica la frecuencia mensual de hembras en el grado I y en la Figura 2 se señala la frecuencia del grado II, puede notarse que, aparentemente en todo el año, a excedción de $\mathrm{Fe}$ brero, Marzo y Mayo la condición de desarrollo es máximo en el res to del año.

El máximo número de especímenes con escaso desarrollo de la masa visceral correspondió al intervalo de $25.1-30.0 \mathrm{~mm}$ y no se repartió ningún ejemplar en esta condición en el intervalo mayor $(40.1-45.0 \mathrm{~mm})$.

En los ejemplares femeninos con gónadas en grado II la mayor frecuencia se encontró en la clase $30.1-35.0 \mathrm{~mm}$ y la mínima en el intervalo máximo $40.1-45.0 \mathrm{~mm}$.

La relación entre los grados es 856 : 340 con predominio significativo de el grado II. En los machos no fue posible cuantificar diferencias en estudios macroscópicos, sin embargo, se determinó que en los especimenes examinados pertenecientes a Septiembre, Diciembre y Marzo los alveolos contenian muchos espermatozoides en el lúmen.

El mayor número (362) correspondió a la talla intermedia $(25.1$ - $30.0 \mathrm{~mm})$ y el minnimo (3) al máximo intervalo (40.1 $45.0 \mathrm{~mm})$.

Las cantidades de espécimenes por sexo, discriminados en fecha y meses de colección se indican en la Tabla II.

En Julio, Agosto y Diciembre de 1974 la cantidad de hembras es mayor, lo mismo ocurre en Enero y Mayo de 1975. En Junio y Abril de 1975 la relación se invierte con una dominancia significativa de los machos.

En Febrero y Marzo de 1975, Septiembre, Octubre y Noviembre de 1974 , la proporción es equilibrada sin dominancia significativa. El número de especímenes en el cual se observaron gametos de ambos sexos fue de 23 , cantidad mínima en relación a los 2391 ejemplares examinados, y se les catalogó de sexo de acuerdo al número de gametos predominantes. Los ejemplares en los cuales no pudo determinarse el sexo fueron considerados como indeterminados, y el total en esta condición fue de 48 (Tab.III).

En la Tabla IV se observa que en las tallas menores (10.1 $20.1 \mathrm{~mm})$ y en la intermedia $(25.1-30.0 \mathrm{~mm})$ hay maior número 


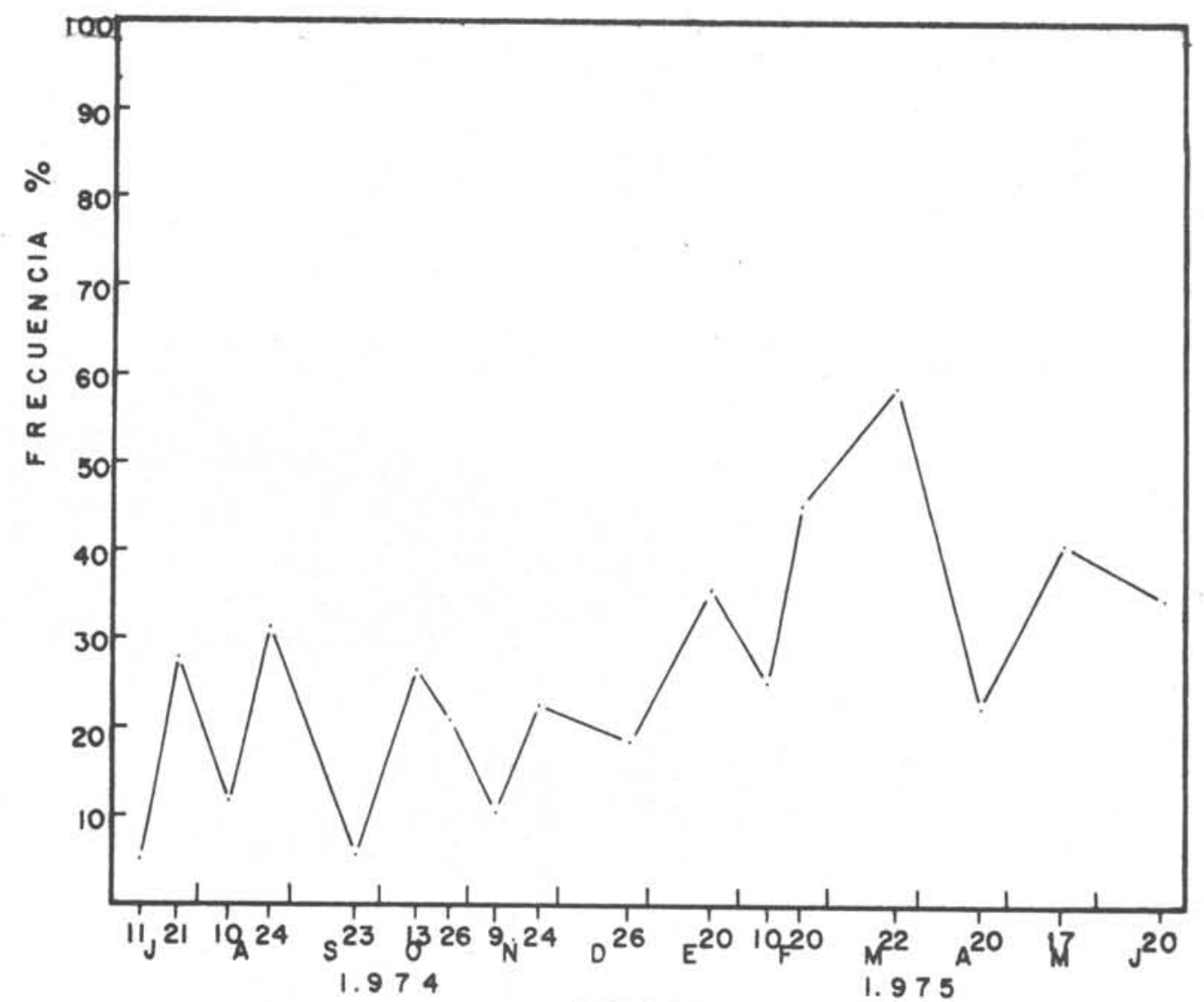

M E SES

Fig. 1. Frecuencia de hembras de Tivela mactroides colectadas en Playa Guiria en el grado de maduración convencional I por fechas de captura durante 1974 y 1975.

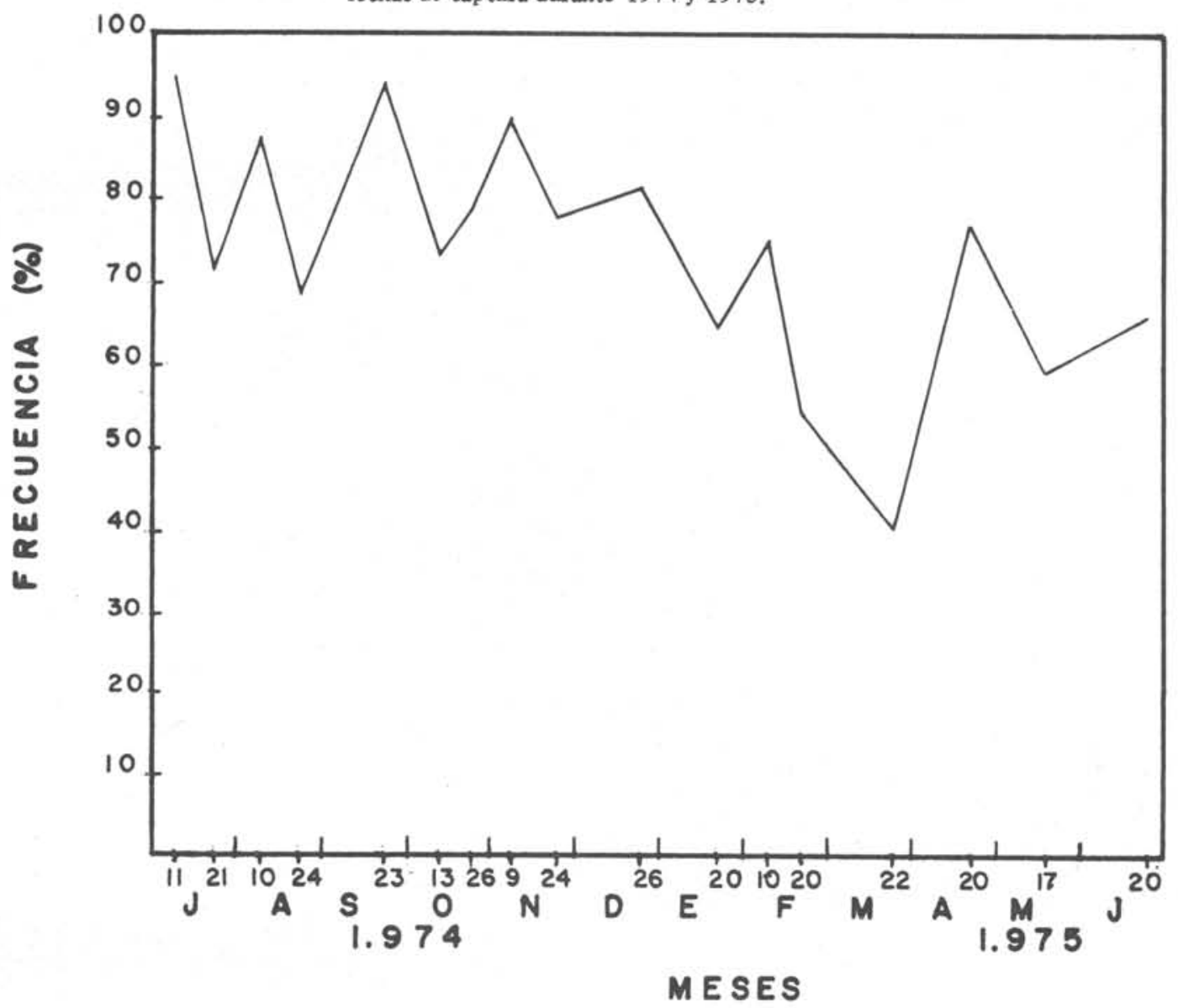

Fig. 2. Frecuencia de hembras de Tivela mactroides colectadas en Playa Guiria en el grado de maduración convencional gonadal II por fechas de capturas durante 1974 y 1975. 
TABLA II. Frecuencias de machos en Tivela mactroides distribuídos por intervalos de tallas de $5 \mathrm{~mm}$ en los meses de colección.

\begin{tabular}{|c|c|c|c|c|c|c|c|c|}
\hline Fecha & $10.1-15.0$ & $15.1-20.0$ & $20.1-25.0$ & $25.1-30.0$ & $30.1-35.0$ & 35.140 .0 & $40.1-45.0$ & Total \\
\hline 11.07 .74 & 0 & 0 & 15 & 19 & 0 & 1 & 0 & 35 \\
\hline 21.07 .74 & 0 & 3 & 1 & 4 & 9 & 2 & 0 & 19 \\
\hline 10.08 .74 & 6 & 17 & 19. & 38 & 30 & 9 & 0 & 119 \\
\hline 24.08 .74 & 0 & 9 & 1 & 9 & 8 & 4 & 0 & 31 \\
\hline 23.09 .74 & 0 & 3 & 3 & 10 & 17 & 19 & 0 & 52 \\
\hline 13.10 .74 & 0 & 2 & 25 & 31 & 20 & 5 & 1 & 84 \\
\hline 26.10 .74 & 0 & 0 & 8 & 16 & 19 & 0 & 0 & 43 \\
\hline 09.11 .74 & 0 & 2 & 16 & 23 & 25 & 4 & 0 & 70 \\
\hline 24.11 .74 & 9 & 30 & 23 & 17 & 9 & 1 & 0 & 89 \\
\hline 26.12 .74 & 0 & 12 & 17 & 17 & 25 & 6 & 0 & 77 \\
\hline 20.01 .75 & 0 & 3 & 25 & 42 & 20 & 12 & 2 & 104 \\
\hline 10.02 .75 & 0 & 3 & 20 & 40 & 35 & 4 & 0 & 102 \\
\hline 20.02 .75 & 0 & 21 & 23 & 28 & 6 & 8 & 0 & 85 \\
\hline 22.03 .75 & 0 & 2 & 0 & .14 & 10 & 0 & 0 & 32 \\
\hline 23.04 .75 & 0 & 12 & 17 & 15 & 14 & 5 & 0 & 63 \\
\hline 17.05 .75 & 0 & 14 & 33 & 23 & 15 & 3 & 0 & 88 \\
\hline 20.06 .75 & 0 & 0 & 5 & 16 & 30 & 2 & 0 & 53 \\
\hline Total & 15 & 133 & 257 & 362 & 292 & 85 & 3 & 1.147 \\
\hline
\end{tabular}

TABLA III

Frecuencias de hembras por estado convencional de maduración y machos de Tivela mactroides examinados durante las fechas de capturas.

\begin{tabular}{|c|c|c|c|c|c|c|}
\hline Fecha & Grado I & Grado II & $\%$ & $\%$ & $\%$ IND & $\begin{array}{l}\text { No Ejem. } \\
\text { Examinados } \\
\end{array}$ \\
\hline 11.07 .74 & 52.63 & 94.061 & 66.66 & 33.33 & 0 & 57 \\
\hline 21.07 .74 & 28.32 & 71.66 & 53.097 & 46.902 & 0 & 113 \\
\hline 10.08 .74 & $12: 49$ & 87.500 & 57.831 & 37.54 & 4.81 & 83 \\
\hline 24.08 .74 & 31.25 & 68.75 & 51.61 & 45.24 & 2.15 & 93 \\
\hline 23.09 .74 & 5.88 & 94.12 & 50.00 & 47.059 & 2.941 & 68 \\
\hline 13.10 .74 & 26.50 & 73.49 & 42.56 & 45.64 & 11.79 & 195 \\
\hline 26.10 .74 & 21.311 & 78.689 & 53.982 & 46.018 & 0 & 113 \\
\hline 09.11 .74 & 10.465 & 89.535 & 40.566 & 56.132 & 3.302 & 212 \\
\hline 24.11 .74 & 22.472 & 77.528 & 51.445 & 48.555 & 0 & 173 \\
\hline 26.12 .74 & 18.74 & 71.26 & 58.00 & 42.00 & 0 & 83 \\
\hline 20.01 .75 & 35.96 & 74.93 & 53.61 & 46.39 & 0 & 166 \\
\hline 10.02 .75 & 25.00 & 75.00 & 50.943 & 48.113 & 0.943 & 212 \\
\hline 20.02 .75 & 45.349 & 54.651 & 50.00 & 50.00 & 0 & 172 \\
\hline 23.03 .75 & 69.09 & 30.91 & 51.16 & 48.48 & 0 & 129 \\
\hline 20.04 .75 & 22.81 & 77.19 & 44.88 & 55.12 & 0 & 127 \\
\hline 17.05 .75 & 41.071 & 58.929 & 56.00 & 44.00 & 0 & 200 \\
\hline 20.06 .75 & 34.940 & 65.000 & 42.56 & 53.53 & 4.10 & 195 \\
\hline
\end{tabular}

TABLA IV.

Distribución obtenida de la proporción de sexos e indeterminados por intervalos de longitud total de 5 mm en Tivela mactroides de Playa Guiria.

\begin{tabular}{|c|c|c|c|c|}
\hline Intervalos & Machos (Total) & Hembras (Total) & Indet. & Total \\
\hline $\begin{array}{l}10.1-15.0 \\
15.1-20.0 \\
20.1-25.0 \\
25.1-30.0 \\
30.1-35.0 \\
35.1-40.0 \\
40.1-45.0\end{array}$ & $\begin{array}{r}15 \\
133 \\
257 \\
362 \\
292 \\
85 \\
3\end{array}$ & $\begin{array}{r}12 \\
100 \\
263 \\
350 \\
362 \\
104 \\
5\end{array}$ & $\begin{array}{r}6 \\
20 \\
13 \\
5 \\
4 \\
0 \\
0\end{array}$ & $\begin{array}{r}33 \\
253 \\
533 \\
717 \\
658 \\
189 \\
8\end{array}$ \\
\hline Total & 1.147 & 1.196 & 48 & 2.391 \\
\hline$\%$ & 47.9716 & $50.0209^{\circ}$ & 2.0075 & 100 \\
\hline
\end{tabular}

de hembras que de machos; lo contrario ocurre en los intervalos mayores de longitud total $(30.1-45.0 \mathrm{~mm})$. Las pruebas de significancia utilizadas sobre los resultados de hembras y machos por separado indican que las frecuencias observadas se desvian muy significativamente (Tab. V).

$\mathrm{Si}$ agrupamos los intervalos de clase combinados de sexos en 3 grupos: de 10.1 a $25.0 \mathrm{~mm}$; de 2,51 a $30.0 \mathrm{~mm}$ y de 30.1 hasta $45.0 \mathrm{~mm}$, se demuestra que en las tallas menores $(10.1-30 \mathrm{~mm})$ hay un equilibrio de sexos pero en tallas mayores de $30.0 \mathrm{~mm}$, la población es dominada por las hembras.
En el estudio histológico de laš gónadas se seleccionaron cinco estados convencionales, atendiendo a las características de los folículos (Tab. VI y VII).

En la Tabla VIII se indican los estados de maduración gonadal de ejemplares adultos.

A pesar del escaso número de animales examinados, es evidente que en ambos sexos son numerosos los estados de desove parcial y maduración total. Esto indica que la evacuación de gametos es seguida del desarrollo de nuevas generaciones de oocitos y espermatocitos a partir de la pared folicular. En hembras con desove to- 
tal y aegeneración puede ocurrir el desarrollo de nuevos foliculos; en Julio, Agosto y Septiembre de 1974 y Febrero de 1975 no se observaron estados avanzados de maduración. En machos, los estados II, III y IV se presentaron en la misma proporción total y solamente se encoptró en Azosto de 1974 un ejemplar en estade indeterminado (I). En Enero de 1975 ce observaron dos machos con folículos evacuados totalmente y signos de degeneración gonadal; esto indica que la actividad gamética es más intensa en machos que en las hembras.

En Febrero de 1975 se determinó un macho con gónadas maduras que presentaba folículos con ovulos residuales atacados por fagocitos; la hembra de menos talla que presentó ovocitos grandes y evidencias de desove midió $11.1 \mathrm{~mm}$.

\section{Discusion}

Los resultados obtenidos en este trabajo nos indican que la especie estudiada se reproduce continuamente todo el año aunque existe mayor predominio de los ovocitos grandes desde Julio a Diciembre de 1974, decreciendo la maduración hasta Marzo de 1975. Esta característica que ha sido sef́alada por diversos investigadores para gran cantidad de invertebrados marinos (Stephenson, 1934 y Vélez, 1975). Los estudios de cortes histológicos revelan que la maduración sexual ocurrió durante todo el affo, aunque se nota un decrecimiento de la actividad gamética a comienzos de 1975 , ya que se observó predominancia de ovocitos pequeños y poco desarrollo gonadal.

Sin embargo, existe una gran variabilidad en los diferentes géneros estudiados hasta ahora en aguas templadas, por ejemplo en Mya arenaria (Coe \& Turner, 1938) y Venus striatula (Ansell, 1961), hay un desarrollo mayor inmediatamente después del desove pero los gametos permanecen en varios estados de maduración desde el otofio hasta el invierno y luego un segundo período gametogénico ocurre en la siguiente primavera, otros bivalvos no maduran tan rapidamente del desove, sino en primavera, esto es válido para Mytilus

T IBLA V.

Resultado de las pruebas de significancia aplicadas a las proporciones de sexos obtenidas. Las frecuencias esperadas fueron calculadas po: ajuste a una distribución normal. P es probabilidad de exceder el valor crítico en las frecuencias observadas entre los diferentes intervalos de talla.

\begin{tabular}{|c|c|c|c|c|c|}
\hline \multirow{2}{*}{$\begin{array}{c}\text { Intervalos } \\
\mathrm{mm}\end{array}$} & \multicolumn{2}{|c|}{ Hembras } & \multicolumn{2}{|c|}{ Machos } & \multirow[b]{2}{*}{$\mathrm{P}$} \\
\hline & Fo & $\mathrm{Fe}$ & Fo & $\mathrm{Fe}$ & \\
\hline $5.1-10.0$ & 0 & 1 & 0 & 2 & \\
\hline $10.1-15.0$ & 12 & 14 & 15 & 21 & 0.436 \\
\hline $15.1-20.0$ & 100 & 88 & 133 & 109 & $0.969 * * *$ \\
\hline $20.1-25.0$ & 263 & 267 & 257 & 285 & 0.207 \\
\hline $25.1-30.0$ & 350 & 401 & 362 & 372 & 0.347 \\
\hline $30.1-35.0$ & 362 & 296 & 292 & 256 & $0.833 * * *$ \\
\hline $35.1-40.0$ & 104 & 108 & 85 & 86 & 0,52 \\
\hline $40.1-45.0$ & 5 & 19 & 3 & 14 & 0.503 \\
\hline $45.1-50.0$ & 0 & 2 & 3 & 2 & \\
\hline Total & 1196 & 1196 & 1147 & 1147 & \\
\hline$x^{2}$ & \multicolumn{2}{|c|}{$36.648 \mathrm{~S}$} & \multicolumn{2}{|c|}{27.7353} & \\
\hline
\end{tabular}

TABLA VI.

Algunas características de los estados de maduración sexual determinados en cortes histológicos de secciones gonadales en hembras de Tivela mactroides.

\begin{tabular}{llll}
\hline \hline Estado & Lumen y contenido folicular & $\begin{array}{l}\text { Tejido conectivo } \\
\text { interfolicular. }\end{array}$ & $\begin{array}{l}\text { Pared y diámetro folicular apro- } \\
\text { ximado }\end{array}$ \\
\hline \hline
\end{tabular}

I

Indeterminado

II

Maduración avanzada

III

Maduración total

IV

Desove parcial
Lumen escaso, puede contener células foliculares y algunas ovogonias con núcleos semicoloreados.
Ausente o escaso

Escaso

Lumen mayor que en 1 , no totalmente lleno de ovocitos pedunculados y pequeños con núcleos vesiculosos.
Pared folicular colapsada, con células germinales en crecimiento; diámetro entre 130 a 250 micras.

Pared folicular expandida con ovocitos en crecimiento; diámetro entre 300 y 700 micras.
Escaso pero menor que en II.
Pared expandida muy desarrollada con células en crecimiento; diámetro oscila entre 500 a $1190 \mathrm{mi}$ cras. canzan 90 micras de longitud, alnos pedunculados.

May or que en el estado anterior.
Pared delgada con sintomas de contracción, pocos ovocitos en crecimierito; diámetro igual que en III.

$$
\text { V }
$$

Desove total y degeneración.
Lumen amplio, no totaimente lleno de ovocitos maduros, alguotros pequeños en crecimiento:
Pared muy delgada, con escasas células en crecimiento y en algunos tiende a fragmentarse; diámetro del grado de degeneración.
Igual que en IV, pero tiende a aumentar al avanzar la desaparición de algunos foli-

culos. miento, algunos maduros en reabsorción. 
TABLA VII.

Algunas características de los estados de maduración sexual determinados en cortes histológicos de secciones gonadales en machos de Tivela mactroides.

\begin{tabular}{|c|c|c|c|}
\hline Estado & Lumen y contenido folicular & $\begin{array}{l}\text { Tejido conectivo } \\
\text { interfolicular }\end{array}$ & $\begin{array}{l}\text { Pared y diámetro folicular apro- } \\
\text { ximado, }\end{array}$ \\
\hline$\frac{\text { I }}{\text { Indeterminado }}$ & $\begin{array}{l}\text { Lumen escaso o colapsado, vacío } \\
\text { o con algunas células al comenzar } \\
\text { la maduración. }\end{array}$ & Abundante & $\begin{array}{l}\text { Pared folicular contraída con al- } \\
\text { gunas células en crecimiento, diá- } \\
\text { metro folicular entre } 130 \text { y } 250 \\
\text { micras. }\end{array}$ \\
\hline $\begin{array}{c}\text { II } \\
\text { Maduración avanzada }\end{array}$ & $\begin{array}{l}\text { Lumen amplio con gran cantidad } \\
\text { de células no maduras (espermato- } \\
\text { citos) y pocas espermatidas y es- } \\
\text { permatozoides; en el centro. }\end{array}$ & $\begin{array}{l}\text { Abundante pero menor } \\
\text { que en el estado ante- } \\
\text { rior. }\end{array}$ & $\begin{array}{l}\text { Pared con muchas células en de- } \\
\text { sarrollo, epitelio en crecimiento; } \\
\text { diámetro entre } 450 \text { y } 800 \text { micras. }\end{array}$ \\
\hline$\stackrel{\text { III }}{\text { N. aduración total }}$ & $\begin{array}{l}\text { Lumen amplio lleno de espermati- } \\
\text { das y espermatocitos cerca de la } \\
\text { pared, los espermas dispuestos } \\
\text { en el centro. }\end{array}$ & $\begin{array}{l}\text { Abundante, igual que } \\
\text { en II. }\end{array}$ & $\begin{array}{l}\text { Pared y epitelio germinal en má- } \\
\text { ximo desarrollo con células en } \\
\text { maduración, diámetro como en II. }\end{array}$ \\
\hline $\begin{array}{c}\text { IV } \\
\text { Desove parcial }\end{array}$ & $\begin{array}{l}\text { Lumen amplio con muchos es- } \\
\text { permatozoides en el centro, algu- } \\
\text { nas veces con restos celulares. }\end{array}$ & $\begin{array}{l}\text { Abundante, igual que } \\
\text { en III. }\end{array}$ & $\begin{array}{l}\text { Pared ligeramente contraída, epi- } \\
\text { telio menos desarrollado que en } \\
\text { III; diámetro entre } 435.2 \text { y } 816 \\
\text { micras. }\end{array}$ \\
\hline $\begin{array}{l}\text { V } \\
\text { Desove total y degene- } \\
\text { racion. }\end{array}$ & $\begin{array}{l}\text { Lumen semivacío, con espermato- } \\
\text { zoides en reabsorción y restos ce- } \\
\text { hulares. }\end{array}$ & $\begin{array}{l}\text { Aumenta al avanzar } \\
\text { la degeneración }\end{array}$ & $\begin{array}{l}\text { Pared delgada con pocas células } \\
\text { en crecimiento; en algunos casos } \\
\text { tiende a fragmentarse. Diámetro } \\
\text { entre } 200 \text { y } 711 \text { micras. }\end{array}$ \\
\hline
\end{tabular}

\title{
TABLA VIII.
}

Estados histológicos de desarrollo gonadal en Tivela mactroides determinados en las muestras mensuales colectadas en Playa Guiria durante 1974 y 1975 .

\author{
1 = Indeterminado \\ II = Maduración avanzada \\ III = Maduración total \\ IV $=$ Desove parcial \\ $\mathrm{V}=$ Desove total $\mathrm{y}$ degeneración
}

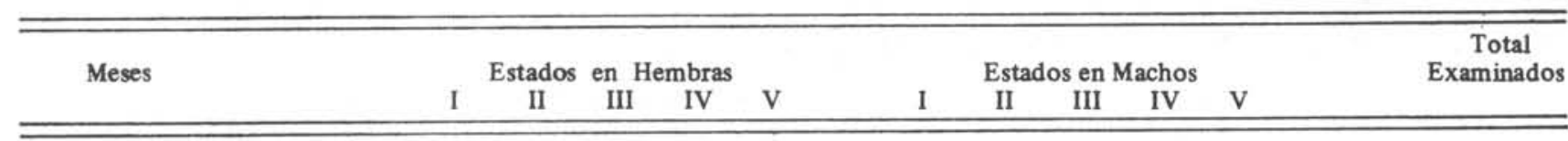

\section{Julio - 74}

Agosto - 74

Septiembre -74

Octubre - 74

Noviembre -74

Diciembre - 74

Enero - 75

Febrero - 75

Marzo - 75

Total

\begin{tabular}{|c|c|c|c|c|c|c|c|c|c|}
\hline & $\begin{array}{l}2 \\
5\end{array}$ & 1 & 2 & 1 & $\begin{array}{l}1 \\
3\end{array}$ & 1 & 1 & & $\begin{array}{r}5 \\
12\end{array}$ \\
\hline & 1 & 4 & 1 & & 2 & 3 & 3 & & 14 \\
\hline 1 & & 3 & & & 3 & 2 & 1 & & 10 \\
\hline 2 & 1 & 3 & 1 & & 3 & 6 & 2 & & 18 \\
\hline 1 & 1 & 4 & & & 1 & 1 & & & 8 \\
\hline 1 & 1 & 2 & 2 & & & 1 & 1 & 2 & 10 \\
\hline & & 2 & 2 & & 1 & 2 & 5 & 1 & 13 \\
\hline 1 & & 3 & 2 & & 1 & 2 & 3 & & 12 \\
\hline 6 & 11 & 22 & 10 & 1 & 15 & 18 & 16 & 3 & 102 \\
\hline
\end{tabular}

edulis y algunas ostras (Loosanoff, 1937), lo que causa una pérdida de la sexualidad de las especies en los períodos en que los tejidos interfoliculares comienzan a acumular reservas.

Boyden (1971). estudiando en forma comparativa la reproducción de Cardium edule y C. glaucum por examen microscópico de frotis gonadal, señala un número muy alto de especímenes indeterminados (30\%). Pósteriormente Kingston (1974) trabajando en las mismas especies pero con examen de cortes histológicos no encontró ejemplares indeterminados.

La proporción de sexos observada en conjunto en Tivelo mactroides no difiere significativamente, pero las frecuencias esperadas no se ajustan a una distribución normal. Es notorio que en los intervalos de talles superiores halla más abundancia de hembras pero no on las tallas juveniles donde al parecer los machos no pare- cn dominar significativamente, podriamos explicar estas variaciones debito a errores ae muestreo o a posibles distribuciones diferenciales causaas por factores no deturminados. Otra explicación podría basarse en un cambio de sexo (inversión sexual) de machos iuveniles a hembras adultas, esto parece lógico pero no se pudo aemostrar, aunque se detectaron algunos ejemplares juveniles ambisexuales, una tercera hipótesis sería aue hubjera una mortalidad de machos jovenes mayor que la de hembras, lo que causaría el aumento de estas. Esta última suposición ha sido demostrada en algunas especies (Donax vitatus. Cardium edule y Mytilus edulis). en donde las hembras tienden a ser más numerosas al aumentar ta edad de la población (Pelsenner, 1926).

Coe (1943) que es el autor que más se dedicó al estudio de la sexualidad en moluscos, basado en estudios realizados en Ttve- 
la stultorum de zonas templadas conceptuó este género como un hermafrodita funcional, con maduración simultánea de gametos de diferentes sexos en uл mismo individuo; esta suposición generalizada queda descartada de plano para Tivela mactroides y aún en el supuesto de que las áreas foliculares indeterminadas representaran potencialmente gónadas de sexo opuesto al que se determinó en los cortes histológicos, más esta posibilidad aunque pudiera contar con el apoyo de los resultados obtenidos sobre proporción de sexos no pudo comprobarse con evidencias directas en cortes histológicos. El ciclo reproductivo de los bivalvos según Boyden (1971) ocurre por un cambio progresivo desde una condición donde la gónada está indiferenciada pasando por un proceso de diferenciación de los gametos hasta la liberación de los mismos, parcial o totalmente, volviendo a los estados iniciales de desarrollo. En lamelibranquios, estos estados han sido muy estudiados y no existe mucha concordancia en la aplicación y separación de las fases en los moluscos estudiados. En Tivela mactroides la maduración sexual se estudió considerando una escala de madurez de 5 rados. 4 correspondientes a adultos y 1 a juveniles pero presente en áreas foliculares de adultos. $\mathrm{El}$ análisis de los cortes histológicos de la gốnada de $T i$ ye'a mactroides reveló también un aumento de los estados de desove (IV y V) entre Enero y Marzo de 1975 (Tab. VIII) que es el período en oue la temperatura y la salinidad alcanzaron los valores más bajos. No creemos que la disponibilidad de alimentos sea limitante en Playa Guíria, ya aue son áreas pesqueras de alta productividad. Es probable que ocurran ciclos gametogénicos de corta duración ya que los estados de regresión folicular no son muy determinantes. No es común encontrar folículos totalmete llenos de ovocitos como se ha señalado para Crassostrea rhizophorae y Mytilus. edulis, especies tropicales. Debido a que el estado dominante es el desove parcial (IV) muchos ovocitos de la pared folicular que no pudieron madurar entran en estado de citolisis y reabsorción (V). El ejemplar hembra de menor talla con signos de evacuación parcial midió $11,1 \mathrm{~mm}$ lo que indica que la especie alcanza el $74.93 \%$ de su tamano total después del primer desove. El hermafroditismo observado en Tivela mactroides en los estudios de frotis fue del $0.97 \%$ distribuidos predominantemente en los juveniles, y por observación de cortes se identificó un macho con un folículo degenerado con residuos de ovocrios, esto plantea un caso de protoginia inverso a la protandria de las ostras. Los estudios de cambios de sexo en bivalvos ha sido muy estudiado en ostras y se ha detectado un hermafroditismo que va aesde el $0.35 \%$ en Crassostrea gigas hasta $3.9 \%$ en $C$. rhizophorae (Vélez, 1975). Lay-Salas (1966) reportó en Eurhomaleo rufa aimeja dioica de Chile unthermafroditismo de $1 \%$ en su mayoría constiturda por ejemplares grandes con gi.netos de ambos sexos. Esto demuestra que el sexo en bivalvos es un fenómeno que debe estudiarse muy cuidadosamente.

\section{Conclusiones}

1. La especie adulta es dioica pero pueden hallarse casos aislados de hermafroditismo. La relación sexual total no difiere de la proporción $1: 1$.

2. Las frecuencias observadas por separado de hembras y machos difieren significativamente de las esperadas, por los que se supone que exisien diferencias de comportamiento ecológico entre los sexos.

3. E1 análisis de la distribución de sexos a diferentes intervalos de tallas revela que entre $10.1-25.0 \mathrm{~mm}$ existe una dominancia no significativa de los machos; entre 25.1 $30.0 \mathrm{~mm}$ hay un equilibrio de la relación sexual y en las tallas máximas 30.1 - $45.0 \mathrm{~mm}$ el número de hembras es significativamente superior.

4. La maduración gamética ocurrí aparentemente todo el año, con mayor intensidad desde Julio hasta Diciembre de 1974 y en Abril de 1975. sin embargo no existe un péríodo donde cese totalmente la reproducción.

5. La obsérvación de los cortes histológicós reveló que existe asincronia en la maduracion sexual de los folfculos de de un mismo especimen, $\mathrm{y}$ el desove ocurre todo $\mathrm{el}$ affo por la repetición contínua de ciclos gametogénicos.

\section{Referências Bibliográfieas}

ANSELL, A. D. 1961. The development of the primary gonad in Venus striatula (da Costa). Proc. malac. Soc. Lond,, 34 (5): 243-247.

BOYDEN, C. R. 1971. A comparative study of the reproductive cycles of the cockles Cerastoderma edule and C. glaucum. J. mar. biol. Ass. U. K., 51:605-622.

CADDY, J. F. 1967. Maduration of gametes and spawning in $M a$ coma balthica (L.). Can. J. Zool., 45 (6): $955-965$.

CARVAJAL, J. R. 1969. Fluctuación mensual de las larvas y crecimiento del meillón. Perna perna y las condiciones ambientales de la Ensenada de Guatapanare, Edo. Sucre, Venezuela, Boln Inst. oceanogr., Univ. Oriente, $8(1 / 2): 13-20$.

COE, W. R. 1943. Sexual differentiation in mollusks. I. Pelecypods. O. Rev. Biol., 18: 154-164.

- - - - \& TURNER, H. J. 1938. Development of the gonads and gametes in the soft-shell clam (Mya arenaria). J. Morph., $62(1): 91-111$.

KINGSTON, P. 1974. Some observations on the effects of temperature and salinity upon the krowth of Cardium edule and Cardium glaucum in the laboratory. J. mar. biol. Ass. U.K., 54 (2): 309-317.

LAY SALAS, J. 1966. Escala de madurez sexual de Eurhomalea rufa (Lamarck, 1818). Estudios oceanol., 2:21-24.

LOOSANOFF, V. L. 1937. Spermatogenesis in the hard-shell clam (Venus mercenaria, Linnaeus). Yale J. Biol. Med., 9 (5) $437-442$.

-_- - 1953. Reproductive cycle in Cyprina islandica Biol. Bull. mar. biol. Lab., Woods Hole, 104: 146-155.

NARCHI, W. 1972. Comparative study of the function morphology of Anomalocardia brasiliana (Gmelin, 1791) and Tivelo mactroides (Born, 1778) (Bivalvia, Veneridae). Bull. mar. Sci., 22 (3): 643-670.

PELSENNER, P. 1926. La proportion sur l'embryologie des gastropodes. Mém. Acad. r. Belg., Cl. Sci., 2(3): 1-167.

STEPHENSON, A. 1934. The breeding of reef animals. Part II. Invertebrates other than corals. Rep. Gt Barriet Ref Comm., $3: 247-272$.

VELEZ, R.A. 1975. Estudio de la reproducción de la ostra de mangle, Crassostrea rhizophorae. Tesis de Grado. Universidad de Oriente, Instituto Oceanográfico.

--- - \& MARTINEZ, R. 1967. Reproducción y desarrollo larval experimental del mejillón comestible de Venezuela, Perna perna (Linnaeus, 1758). Boln Inst. oceanogr., Univ. Oriente, $6(2): 263-285$.

WARMKE, G. \& ABBOTT, R. T. 1961. Caribbean seashells. Narberth, Livingston, $346 \mathrm{p}$.

YOLOYE, V. 1970. The sexual phases of the "West African bloody cockle" Anadara senilis (Linnaeus), (Mollusca:Bivalvia), Ve. liger, 17 (1): 70-72. 